\title{
Cloning and molecular analysis of voraxin- $\alpha$ gene of Rhipicephalus (Boophilus) microplus
}

\author{
Binod Kumar • Srikanta Ghosh
}

Received: 13 January 2014/ Accepted: 9 May 2014/Published online: 25 May 2014

(c) Indian Society for Parasitology 2014

\begin{abstract}
To identify suitable targets for development of cross-protective tick vaccine, in silico analysis was attempted and male tick derived molecule, voraxin- $\alpha$ was targeted. The voraxin- $\alpha$ homologue of Rhipicephalus (Boophilus) microplus was cloned, sequenced and analyzed employing standard methods. The deduced amino acids sequence analysis of the 419 bp cloned voraxin- $\alpha$ gene of $R$. (B.) microplus indicated very high (94.6\%) similarity with voraxin- $\alpha$ of the $R$. appendiculatus and moderate to low identity with Amblyomma hebraeum, Dermacentor silvarum and Haemaphysalis longicornis. The results suggest that recombinant voraxin- $\alpha$ might be a good candidate as cross-protective anti-tick vaccine.
\end{abstract}

Keywords Voraxin- $\alpha \cdot$ Anti-tick vaccine

Rhipicephalus (Boophilus) microplus

\section{Introduction}

Ticks as veterinary parasite, cause deleterious effects in two ways, firstly, they suck significant quantities of blood, which itself, affect the weight gain and milk production of dairy animals (Graf et al. 2004). Secondly, the impact of tick borne diseases is immense on livestock health (de la

\section{B. Kumar $\cdot$ S. Ghosh $(\varangle)$}

Entomology Laboratory, Division of Parasitology, Indian

Veterinary Research Institute, Izatnagar 243122, India

e-mail:sshoshp@yahoo.co.in

\section{Present Address:}

\section{B. Kumar}

Department of Veterinary Parasitology, College of Veterinary

Science and Animal Husbandry, Junagadh Agricultural

University, Junagadh 362001, India
Fuente and Kocan 2003). Amongst the different species of ticks, Rhipicephalus (Boophilus) microplus is a major one affecting livestock health, as a debilitating agent itself and as a vector of babesiosis and anaplasmosis (Sonenshine 1993). On global basis the losses incurred by livestock industry due to ticks and tick-borne diseases (TTBDs) was estimated in the range of 14,000-18,000 million US\$/year (De Castro 1997). In India, the annual cost of control of TTBDs of cattle has been estimated as US\$498.7 million (Minjauw and Mc Leod 2003).

The TTBDs control program is solely focused on repeated use of harmful chemicals which is not eco-friendly and pose serious threat to environment. There is also increasing evidence that present strategies based on acaricides are not cost effective (Pegram et al. 1993; Ostfeld et al. 2006). Regardless of the demonstrated success of acaricides, societal and scientific concerns over the exclusive dependency upon chemicals have emphasized the need for the development and introduction of alternatives to acaricides that are consistent with the principles of sustainable TTBDs control (Willadsen 2006; Madzimure et al. 2011).

Recent development of a generic approach for the control of ticks is the use of the anti-tick vaccine, which is designed to protect host animals from tick infestations (Ghosh et al. 2007; Kumar et al. 2012). Vaccine-controlled field trials in combination with acaricide treatments demonstrated that an integrated approach resulted in control of tick infestations while reducing the use of acaricides (de la Fuente and Kocan 2003; de la Fuente et al. 2007). These trials demonstrated that control of ticks by vaccination has the advantages of being cost-effective, reducing environmental contamination, and preventing the selection of drug resistant ticks that result from repeated acaricide application. In addition, these vaccines may also prevent or reduce 
transmission of pathogens by reducing tick populations and/or affecting vectorial capacity (de la Fuente et al. 2007; Rodriguez et al. Rodriguez Valle et al. 2004).

Though, the large number of antigens were investigated for their effects against ticks, the only antigen i.e., Bm86 has been transformed into the commercial vaccine. The Bm86 molecule derived from the $R$. (B.) microplus showed variable efficacy against different strains of the homologous and a few heterologous species (Garcia-Garcia et al. 1999; Sossai et al. 2005). There is an urgent need of identification of conserved vital tick molecules which may be exploited for the development of vaccine against multiple tick species. The vital organ and its secretory and regulatory molecules may be targeted for the development of eco-friendly sustainable cross protective anti-tick vaccine. Recent data indicated that the host immunoglobulin can cross the gut of the ticks to reach to hemolymph to cells (Vaughan et al. 2002; de la Fuente et al. 2011). The finding has increased the possibilities of targeting both intracellular and secretory molecules for vaccine development.

Since copulation of female Ixodid ticks is mandatory in order to achieve full engorgement and production of fertilized eggs, mating-related molecules are important in the tick feeding and reproductive behaviour (Kaufman 2007). Weiss and Kaufman (2004) cloned male testis/vas deferens-derived 'voraxin' from Amblyomma hebraeum and first showed that injection of recombinant voraxin into virgin females stimulates blood feeding to engorge. In addition, mean tick weight was significantly reduced and $75 \%$ of the mated female ticks tested did not engorge at all on immunized animals. Recently, Yamada et al. (2009) cloned and characterized complete cds of voraxin- $\alpha$ homologue of $R$. appendiculatus and following immunization $40 \%$ reduction in engorgement and $50 \%$ reduction in egg weight upon homologous challenge infestations was recorded. These results suggest the feasibility of voraxin- $\alpha$ as an useful anti-tick antigen. Until now, there is no information on voraxin- $\alpha$ homologues in $R$. (B.) microplus. In this study, we report the cloning of voraxin- $\alpha$ homologue gene of $R$. (B.) microplus and analysis of genetic similarity or dissimilarity with other voraxin- $\alpha$ homologue from different tick species. This in silico study will help in decision making process to consider this molecule as broad-spectrum anti-tick vaccine candidate before going for more expensive and complicated vaccination trial.

\section{Materials and methods}

\section{Ticks}

Rhipicephalus (Boophilus) microplus ticks (IVRI line I, registration number: NBAII/BM/1/1998) was used for the study. The reference tick line was maintained as reported by Ghosh and Azhahianambi (2007). Briefly, the ticks were fed on healthy calves of 4-6 months old which were maintained as per the guideline of Committee for the Purpose of Control and Supervision of Experiments on Animals (CPCSEA), a statutory Indian body monitoring the use of animals in experimentation. Usually 2-3 calves were used for each feeding cycle. After each feeding cycle, the animals were kept free for 15-20 days. Fully engorged adults were kept in desiccators where 85-90\% relative humidity (RH) was maintained. The desiccators were kept at $28{ }^{\circ} \mathrm{C}$ for oviposition. After completion of oviposition, the dead female ticks were removed from the glass tubes in order to avoid the fungal growth. The freshly laid eggs were kept at $28{ }^{\circ} \mathrm{C}$ with $85-90 \% \mathrm{RH}$ for maturation and hatching to larvae. The homogeneity in the reference lines was periodically checked after cloning and sequencing of its 16S rRNA gene (Accession Nos. GU222462, GU323287, GU323288).

RNA extraction, cDNA synthesis and cloning of voraxin- $\alpha$ gene

Four days old partially fed male and female ticks were used for the isolation of total RNA using TRIZOL reagent (Invitrogen, Carlsbad, CA, USA) according to manufacture's protocol. Reverse transcription was performed using the first strand cDNA synthesis kit (Fermentas, USA).

For cloning of the gene, different sets of primers were designed from the conserved regions of voraxin- $\alpha$ homologue of Amblyomma hebraeum (AB488697), Rhipicephalus appendiculatus (AY442319), Dermacentor silvarum (HQ908089) and Haemaphysalis longicornis (HQ908090). Different permutations and combinations were attempted to optimize the reagents and PCR condition for amplification of voraxin- $\alpha$ homologue of $R$. (B.) microplus. PCR fragments, which are within the expected size for the voraxin- $\alpha$, were purified and routinely cloned into TA-cloning vector (Ins TAclone ${ }^{\mathrm{TM}}$ PCR Cloning Kit, Fermentas, USA) and colony PCR positive clone was sequenced.

\section{Sequence analysis}

Nucleotide sequence identity analysis was performed using the BLAST program (NCBI). All nucleotides [open reading frame (orf) only] and its deduced amino acid sequences from the different species of ticks were analyzed using the program MegAlign (DNAstar, USA). The deduced amino acid sequences were used to seek for orthologs in the nonredundant GenBank protein sequence database by BLASTP analysis. The generated sequence of $R$. (B.) $m i$ croplus and retrieved sequences of other hard ticks $[A$. hebraeum (AB488697), R. appendiculatus (AY442319), D. silvarum (HQ908089) and H. longicornis (HQ908090), 
parentheses contain GenBank accession number] were aligned using ClustalW, neighbour-joining analysis was performed using the Mega4 package (Tamura et al. 2007). Gaps were treated as pair wise deletions, amino acid distances were calculated using Poisson model and branch supports were estimated using bootstraps analysis $(10,000$ bootstraps).

\section{Results}

Amplification of voraxin- $\alpha$ gene of Rhipicephalus (Boophilus) microplus

The voraxin- $\alpha$ gene was amplified using the primer set (forward primer: $5^{\prime}$ ACGTCACCCATGTTGATC $3^{\prime}$ and reverse primer: 5'GATGGCAGTCCCTTGCG3') without any nonspecific reaction using the optimized PCR condition: initial denaturation of $95{ }^{\circ} \mathrm{C}$ for $2 \mathrm{~min}$ and 32 cycle of $94{ }^{\circ} \mathrm{C}$ for $40 \mathrm{~s}, 48{ }^{\circ} \mathrm{C}$ for $30 \mathrm{~s}$ and $72{ }^{\circ} \mathrm{C}$ for $30 \mathrm{~s}$ and final extension at $72{ }^{\circ} \mathrm{C}$ for $15 \mathrm{~min}$. The partial cds (incomplete $3^{\prime}$ ends) of the gene of $R$. (B.) microplus was amplified as a 419 bp product (Fig. 1A). The GenBank accession numbers of the cloned gene is JX502818. The nucleotide sequence was 419 in length, including nine nucleotides upstream of the initial ATG codon and the $3^{\prime}$ end of the gene was incomplete (Fig. 1B).

\section{Sequence analysis}

BLASTP analysis of the submitted voraxin- $\alpha$ sequence using non-redundant GenBank protein database retrieved the same protein. Alignments of deduce amino acid sequences of voraxin- $\alpha$ gene from different hard ticks were shown in Fig. 1C. The variation in amino acid sequences among the tick species which is highlighted (black shadow) and the percentage identity amongst ticks were in the range of 17.6-90.4\% (Table 1). The per cent identities of nucleotide sequence of voraxin- $\alpha$ gene of $R$. (B.) microplus were 90.2, 42.7, 39.5 and $42.9 \%$ against $R$. appendiculatus, $D$. silvarum, $H$. longicornius and A. hebraeum, respectively (Table 1).

Phylogenetic analysis of the amino acid sequences is shown in Fig. 1D. On the basis of amino acid composition of voraxin- $\alpha$, the $R$. (B.) microplus and $R$. appendiculatus comes within same clade which once again confirm the Rhipicephalus species were paraphyletic with respect to the species of Boophilus.

\section{Discussion}

The development of new methods to control tick infestations and reduce the incidence of tick borne diseases while minimizing acaricide applications is essential towards improving cattle health and production in tropical and subtropical regions of the world. Development of vaccine against tick species with broad spectrum activity require the discovery and characterization of new tick protective antigens, a process that constitutes the limiting steps for advancing this area of research (Canales et al. 2009). Antitick vaccines have been developed based on the molecular cloning, characterization and analysis of tick molecules that play an important role in tick physiology. Weiss and Kaufman (2004) reported that the transfer of voraxin- $\alpha$ and voraxin- $\beta$ via copulation is essential for female engorgement and oviposition in A. hebraeum. He also speculated that male factor (MF) from male gonad of A. hebaerum, which can hasten the onset of salivary gland degeneration (Lomas and Kaufman 1992) and engorgement factor (EF) from D. variabilis, which are produced by the male and promotes female feeding to repletion (Pappas and Oliver 1972) are the same substance because both factors are found in testis of fed males. Fortunately, the recombinant voraxin- $\alpha$ showed both MF and EF activity. Yamada et al. (2009) cloned and characterized the complete cds of voraxin- $\alpha$ homologue of $R$. appendiculatus and reported that voraxin- $\alpha$ is not only expressed in male gonad but also ubiquitously expressed in virgin and mated females and engorging nymphs. But the level of expression of voraxin$\alpha$ in nymphs and in females were very-very low (i.e. $1 / 1,000$ or less per actin) compared to those in male testis. In the present study we amplified for the first time the voraxin- $\alpha$ gene from both male and females of $R$. (B.) microplus.

Analysis of nucleotide sequence (orf only) and the deduced amino acid sequence of the cloned voraxin- $\alpha$ cDNA showed high level of conservation compared to the voraxin- $\alpha$ sequence from $R$. appendiculatus whereas, the variation among the different other ticks were moderate to high. The predicted amino acid similarities give the hypothesis that voraxin- $\alpha$ is widely conserved in different tick genera. Moreover, amplification of voraxin- $\alpha$ from female tick RNA signaled towards possible role of voraxin$\alpha$ in female tick physiology (Yamada et al. 2009).

The voraxin- $\alpha$, which is classified as concealed antigen elicit protective anti-tick immune response in rabbit when immunized with recombinant voraxin- $\alpha$. Immunization trial showed $40 \%$ reduction in female tick weight and $50 \%$ reduction in egg weight upon homologous challenge infestation of $R$. appendiculatus in rabbit (Yamada et al. 2009). Very high level of similarity $(94.6 \%)$ in amino acid sequence and sharing of common clade in phylogenetic analysis for voraxin- $\alpha$ homologue of $R$. appendiculatus and $R$. (B.) microplus creates a possibility of development of cross-protective vaccine against these two species. This preliminary work suggest that recombinant voraxin- $\alpha$ might be a good candidate as cross-protective anti-tick vaccine. 

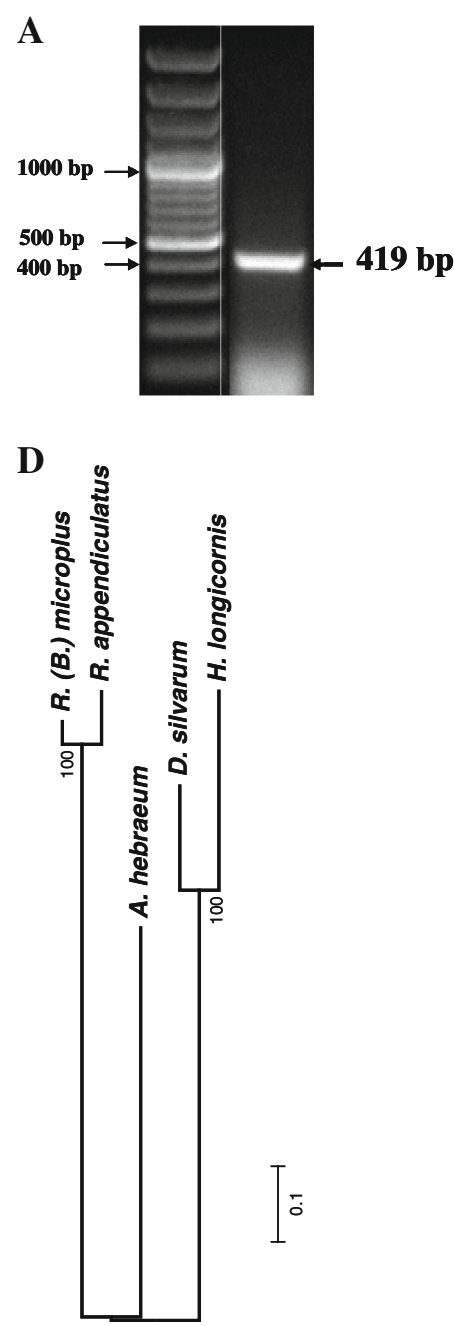

Fig. 1 Amplification and molecular analysis of voraxin- $\alpha$ gene. $\mathbf{A}$ and B: PCR amplification and nucleotide sequence of voraxin- $\alpha$ homologue of $R$. (B.) microplus (419 bp), respectively. DNA ladder: 100 bp plus DNA ladder (MBI, Fermentas, USA). C and D: Sequence alignment and neighbour-joining analysis of deduce amino acid

C
R. (B.) microplus

R. appendiculatus

D. silvarum

A. hebraeum

R. (B.) microplus

R. appendiculatus

D. silvarum

H. longicornis

A. hebraeum

R. (B.) microplus

$R$. appendiculatus

D. silvarum

$\mathrm{H}$. longicornis

A. hebraeum

R. (B.) microplus

R. appendiculatus

D. silvarum

$H$. longicornis

A. hebraeum
H. longicornis

\begin{tabular}{lllllll} 
B & \multicolumn{2}{c}{ Start codon } & & & \\
1 & 5'ACGTCACCCA & TGTTGATCGC & CAAGGATCTG & ATGCAGAGTG & GCGGGGGAAA & CAAGACGTAT \\
61 & TGCATCAGCA & TCAATCTGGC & TGTGCTCAAG & TTCACCACCA & ACGCCGGCAG & CCTCAGCTTC \\
121 & GCAAACAGCA & GCCACTGCGA & CTCGGAGGAC & GAGGTCGCCT & ACTCCGAGCT & GTGCCAGATG \\
181 & AGCAATGCAG & TTCATGTCTA & TGATATGAAC & TGGATGACTG & CGTCGCTCAA & GGCCAGTAGG \\
241 & AAGCTCTACA & CCTTCGAGAA & GGCCGAGATG & TTGCTTTCAA & AGGTTCTATT & CCTAAAGGCA \\
301 & TGGTTCCCGA & GCCTCTGCGT & TGCCGCCTTC & CACGCCGAGC & TTGACACTGT & CGACGGAGAA \\
361 & GCCTGCCCAC & AGTCCGAACA & GTACGCAAGA & CTGCTCAGCG & TTCGCAAGGG & ACTGCCATC...3' \\
\hline
\end{tabular}

MLI AKDLMQSGGGNKTYCI SI NLAVLKFTTNAGSLSFANS 40 MLI AKDL MQRGADNKTYCI SI NL AVLKFATSAGSL SLTNG 40 ML I AKDL MHKGTENKTYCI SI NL AVL KFATSADSLADGSH 40 ML I TKELI QKGMENKTFCVSLNL AVL KFATHATSAARGGS 40 ML I TKDL MQKSTENKTFCI SI NL AVL KFATDAGNPGDRCD 40

SHCDSEDEVAYSEL CQMSNAVHVYDMNUMT ASL KASRKLY 80 SHCDSEDEVAYSEVCQMSNAVPVYDMNUMT ASLKASMKLY 80 CDSEDEVAYSEVCQRNNAI P VYDMNWMT ASL KDSKKL YTF 80 CDSEAEVGYSEVCRVHNKVPVFDMNMMT ASL SDSKQL YTF 80 SEDEVAYSEVCQL NSAVPVYDMNMMT ASLSDSRQFYTFEK 80

TFEKAEML LSKVLFLKAWFPSL CVAAFHAELDTVDGEACP 120 TFEKAEML L SKVLFLKAWFPSL CVAAFHAELDTVDREACP 120 EKAEMLLSKVLFLKAWFPSL CVAAFHAELDAVDREACPQS 120 EKAEML LSKVLFLKAWFPPLCI ATFHTELDTVDATQCPHS 120 AEMLL SKVLFLKAWFPSL CVATFHAELDT GR.

QSEQYARLLSVRKGLP

QSEQYARLLSVRKGLDSAVHGESHURRDATDGA.

EQYARL LSVRRGL DSSVHGESHWRRNAADGA.

AQYERL LAVRKAL DAAVHEGNRPWRNYAEDGA.
112

Table 1 The percentage identity of nucleotide (bold letter) and amino acid (italics letter) sequence of voraxin- $\alpha$ homologue (orf only) of different hard ticks

sequences of voraxin- $\alpha$ (orf only) gene of different species of hard ticks, respectively. The amino acid residues that differ from $R$. (B.) microplus are highlighted by black solid shade. The evolutionary distances were computed using the Poisson correction method. Branch value indicated in 0.1 point scale

\begin{tabular}{llllll}
\hline & R. (B.) microplus & R. appendiculatus & D. silvarum & H. longicornis & A. hebraeum \\
\hline R. (B.) microplus & - & $\mathbf{9 0 . 2}$ & $\mathbf{4 2 . 7}$ & $\mathbf{3 9 . 5}$ & $\mathbf{4 2 . 9}$ \\
R. appendiculatus & 90.4 & - & $\mathbf{4 1 . 2}$ & $\mathbf{3 8 . 6}$ & $\mathbf{4 3 . 5}$ \\
D. silvarum & 22.8 & 21.7 & - & $\mathbf{7 1 . 7}$ & $\mathbf{4 4 . 6}$ \\
H. longicornis & 19.9 & 17.6 & 66.4 & - & $\mathbf{4 0 . 8}$ \\
A. hebraeum & 25.9 & 26.8 & 27.7 & 26.8 & - \\
\hline
\end{tabular}

Acknowledgments We thank director of IVRI for providing research facilities. Senior author is highly thankful to the Indian Council of Agriculture Research (ICAR) for providing Senior Research fellowship. The technical support provided by the laboratory staff (Mr. Naresh Kumar) is highly acknowledged.

Conflict of interest We declare that we have no conflict of interest.

\section{References}

Canales M, Almazan C, Naranjo V, Jongejan F, de la Fuente J (2009) Vaccination with recombinant Boophilus annulatus Bm86 ortholog protein, Ba86, protects cattle against $B$. annulatus and B. microplus infestations. BMC Biotechnol 9:29-35 
De Castro JJ (1997) Sustainable tick and tick-borne diseases control in livestock improvement in developing countries. Vet Parasitol 71:77-97

de la Fuente J, Kocan KM (2003) Advances in the identification of protective antigens for recombinant vaccines against tick infestations. Expert Rev Vaccines 2:583-593

de la Fuente J, Almazan C, Canales M, de la Lastra JM, Kocan KM, Willadsen P (2007) A ten-year review of commercial vaccine performance for control of tick infestations on cattle. Anim Health Res Rev 8:23-28

de la Fuente J, Moreno-Cid JA, Canales M, Villar M, Lastra JMP, Kocan KM (2011) Targeting arthropod subolesin/akirin for the development of a universal vaccine for control of vector infestations and pathogen transmission. Vet Parasitol 181:17-22

Garcia-Garcia JEC, Gonzalez IL, Gonzalez DM, Valdes M, Mendez L, Lamberti J (1999) Sequence variations in the Boophilus microplus $\mathrm{Bm} 86$ locus and implications for immunoprotection in cattle vaccinated with this antigen. Exp Appl Acarol 23:883-895

Ghosh S, Azhahianambi P (2007) Laboratory rearing of Theileria annulata-free Hyalomma anatolicum anatolicum ticks. Exp Appl Acarol 43:137-146

Ghosh S, Azhahianambi P, Yadav MP (2007) Upcoming and future strategies of tick control: a review. J Vector Borne Dis 44:79-89

Graf JF, Gogolewski R, Leach-Bing N, Sabatini GA, Molento MB, Bordin EL (2004) Tick control: an industry point of view. Parasitology 129:S427-S442

Kaufman WR (2007) Gluttony and sex in female ixodid ticks: how do they compare to other blood-sucking arthropods? Insect Physiol 53:264-273

Kumar B, Azhahianambi P, Ray DD, Chaudhuri P, de la Fuente J, Kumar R, Ghosh S (2012) Comparative efficacy of rHaa86 and rBm86 against Hyalomma anatolicum anatolicum and Rhipicephalus (Boophilus) microplus. Parasite Immunol 34:297-301

Lomas LO, Kaufman WR (1992) The influence of a factor from the male genital tract on salivary gland degeneration in the female ixodid tick, Amblyomma hebraeum. J Insect Physiol 38:595-601

Madzimure J, Nyahangare ET, Hamudikuwanda H, Hove T, Stevenson PC, Belmain SR (2011) Acaricidal efficacy against cattle ticks and acute oral toxicity of Lippia javanica (Burm F.) Spreng. Trop Anim Hlth Prod 43:481-489

Minjauw L, Mc Leod A (2003) Tick borne diseases and poverty. The impact of tick and tick borne diseases on the livelihoods of small-scale and marginal livestock owners in India and eastern and southern Africa. Research report, DFID animal health programme, Centre for Tropical Veterinary Medicine, University of Edinburgh, UK

Ostfeld RS, Price A, Hornbostel VL, Benjamin MA, Keesing F (2006) Controlling ticks and tick-borne zoonoses with biological and chemical agents. Bioscience 56:383-394

Pappas PJ, Oliver JH Jr (1972) Reproduction in ticks (Acari: ixodoidea). 2. Analysis of the stimulus for rapid and complete feeding of female Dermacentor variabilis (Say). J Med Entomol 9:47-50

Pegram RG, Tatchell RJ, Castro JJ (1993) Tick control: new concepts. World Anim Rev 74(75):2-11

Rodriguez Valle M, Mendez L, Valdez M, Redondo M, Espinosa CM, Vargas M (2004) Integrated control of Rhipicephalus (Boophilus) microplus ticks in Cuba based on vaccination with the antitick vaccine Gavac. Exp Appl Acarol 34:375-382

Sonenshine D (1993) The biology of ticks, vol 2. Oxford University Press, Oxford

Sossai S, Peconick AP, Sales-Junior PA, Marcelino FC, Vargas MI, Neves ES (2005) Polymorphism of the bm86 gene in South American strain of the cattle tick Boophilus microplus. Exp Appl Acarol 37:199-214

Tamura K, Dudley J, Nei M, Kumar S (2007) MEGA4: molecular evolutionary genetics analysis (MEGA) software version 4.0. Mol Biol Evol 24:1596-1599

Vaughan JA, Sonenshine DE, Azad AF (2002) Kinetics of ingested host immunoglobulin $\mathrm{G}$ in hemolymph and whole body homogenates during nymphal development of Dermacentor variabilis and Ixodes scapularis ticks (Acari: ixodidae). Exp Appl Acarol 27:329-340

Weiss BL, Kaufman WR (2004) Two feeding-induced proteins from the male gonad trigger engorgement of the female tick, Amblyomma hebraeum. Proc Natl Acad Sci USA 101: 5874-5879

Willadsen P (2006) Tick control: thoughts on a research agenda. Vet Parasitol 138:161-168

Yamada S, Konnai S, Imamura S, Ito T, Onuma M, Ohashi K (2009) Cloning and characterization of Rhipicephalus appendiculatus voraxin- $\alpha$ and its effect as anti-tick vaccine. Vaccine 27: $5989-5997$ 